\title{
CONTINUOUS VERSUS PULSED PURE-TONE AUDIOMETRY IN A GROUP OF SCHOOL-AGED CHILDREN
}

Catherine van Dijk* and Naeema Osman Department of Communication Pathology, University of Pretoria, South Africa

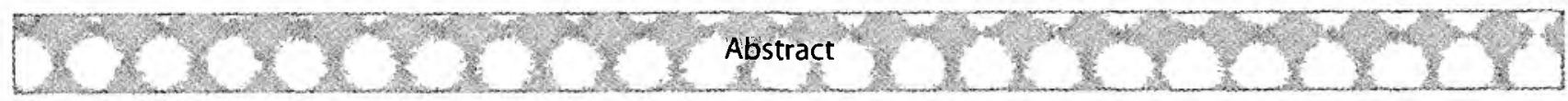

Pure-tone testing is the primary audiological test procedure for the differential diagnosis of hearing loss and hearing disordersin school-agedchildren. Noresearch is currentlyavailable internationally forchildren'sresponses to continuous versus pulsed pure-tones. The aim of this exploratory investigation was to compare the performance of a group of school-aged children on continuous versus pulsed pure-tone audiometry. The aims were to determine whether a threshold difference existed between continuous versus pulsed pure-tones and to record whether a listener preference existed between continuous versus pulsed tones for the frequency range of 125 to $8000 \mathrm{~Hz}$. Eighteen children ( 36 ears) aged between $8-12$ years, participated in a hearing evaluation as well as in a brief three-question interview. Descriptive statistics viz. average threshold,meandifferenceandstandard deviation of thresholdswereused toanalysedata. Listeners'perceivedpreferences werecalculated in percentages and reasonsfor preferringonesignaloveranotherwasanalysedqualitatively. Althoughthe automatically pulsed tone threshold (averaged across the frequencies tested) was lower than for the continuous tone, the difference was only $0.2 \mathrm{~dB}$ in the left ear and $0.5 \mathrm{~dB}$ in the right ear. This small difference is not important in clinical applications for which $5 \mathrm{~dB}$ increments are used in pure-tone audiometry. Where alistener preference was indicated, however, the continuous tones were preferred over pulsed tones by $56 \%$ percent of subjects. These findings differ from similar studies involving adults. This revealed that children may yield different preferences during pure-tone testing than adults and that these preferences should be taken into consideration during testing.

Keywords: audiometry, continuous pure-tone, listener preference, pulsed pure-tone, pure-tone testing, school-aged children, stimulus signal, threshold difference.

$\mathrm{T}$ There are two methods of presentation of pure-tones, i.e. continuous tones and pulsed tones. Pure-tone threshold measurement is a fundamental diagnostic tool in hearing evaluations (DiGiovanni \& Repka, 2007). Pure-tone tests of hearing, using audiometers, have been in use for nearly one hundred years and are quantifiable electronic extensions of the same concepts developed in tuning fork tests (Martin \& Clark, 2003). It is the primary audiologic test procedure for the differential diagnosis of hearing loss and hearing disorders in school-aged children (Blandy \& Lutman, 2005). In addition, pure-tone threshold audiometry has become the standard behavioural procedure for describing auditory sensitivity (Margolis \& Saly, 2007). In 1978, the American Speech-Language-Hearing Association (ASHA) developed clinical guidelines referred to as Guidelines for Manual Pure-Tone Audiometry (ASHA, 1978). In these guidelines they recommended the use of a one to two second sustained tone for threshold determination. This is referred to as a continuous tone. According to an American. survey of audiometric practices two decades ago, the majority of audiologists surveyed, used a manual presentation of continuous tones (Martin \& Sides, 1985). Although the ASHA guidelines (ASHA, 1978) recommend use of a continuous tone, a substitution of pulsed tones is also permitted by ASHA. A pulsed tone can be presented either manually or can be automatically generated by the audiometer. A manually presented pulsed tone would require the tester to present two consecutive tones with a short quiet interval be- tween the two tones. This interval should be one second or shorter in duration (ASHA, 1978).

Studies on children's performance on and preference for continuous versus pulsed pure-tone audiometry is lacking. A study conducted by Burk and Wiley (2004) on women aged 19 years to 44 years showed that pulsed tones are clearly advantageous in presenting what is perceived as an easier task. For many adult clients the pulsed tone is perceived as easier to hear at near threshold levels (Newby \& Popelka, 1985). Other published data suggests that using pulsed tones for adults having tinnitus may result in fewer false-positive responses (30\% less than for continuous tones) and therefore may reduce the number of presentations required to determine the threshold, resulting in more efficient audiological threshold evaluations (Mineau \& Schlauch, 1997).

There are selected studies that support the use of pulsed tones rather than continuous tones in manual threshold audiometry for adults (Dancer \& Conn, 1983; Dancer, Ventry \& Hill, 1976; Gardner, 1947; Hochberg \&Waltzman, 1972; Hood, 1955; Mineau \& Schlauch, 1997). Firstly, in compar-
Autbor Contact:
Department of Communication Pathology
University of Pretoria 0002
South Africa
Fax: +27124203517
Tel: +27124202357
E-mail:Catherine.VanDijk@up.ac.za 
ison to continuous tones, the use of pulsed tones has been shown to decrease the number of false-positives in both normal-hearing listeners and listeners with sensorineural hearing loss with or without tinnitus (Dancer \& Conn, 1983; Dancer et al, 1976; Mineau \& Schlauch, 1997). Secondly, listeners with normal hearing and those with sensorineural hearing loss and tinnitus preferred pulsed tones to continuous tones as a listening task (Gardner, 1947; Hochberg \& Waltzman, 1972). The use of pulsed tones in Bekesy audiometry has also provided insight into using pulsed tones in conventional audiometry (Jerger, 1960). In threshold applications of Bekesy audiometry, pulsed tones are typically presented to minimize the effects of auditory adaptation to a continuous tone (Hallpike \& Hood, 1951; Jerger, 1960; Reger, 1970; Silman \& Silverman, 1991; Sorensen, 1962). In addition, if a 200-milliseconds-on, 200-milliseconds-off pulsed tone is used for threshold assessment, periodic suprathreshold presentations of the test tone acts as a cue for the signal frequency, thereby refreshing the client's memory for the test stimulus (Mineau \& Schlauch, 1997). Furthermore, McCommons and Hodge (1969) found that the pulsed tones used in Bekesy audiometry provide more feedback or cues by which the listener can make a decision regarding the presence of the signal.

The slight preference for pulsed tones in normal-hearing listeners (Burk \& Wiley, 2004), coupled with previous reports demonstrating the benefits of using pulsed tones in threshold assessment for listeners with sensorineural hearing loss and tinnitus (Hochberg \& Waltzman, 1972; Mineau \& Schlauch, 1997), supports the general use of pulsed tones in audiometry. However, in practice it may seem that not all audiologists use this technique. From personal observations, South African audiologists often deviate from standard testing procedures and protocols conveyed to them by their undergraduate training institutions. A diversity of testing techniques and methods are often applied by audiologists in practice and may be due to, amongst others, their employer's personal preferred method of testing that has been imparted to them. The type of test signal (continuous or pulsed) used in pure-tone audiometry may affect the accuracy and reliability of pure-tone thresholds. Some data regarding adults' responses to continuous and pulsed pure-tone audiometry is available (for example: Burk \& Wiley, 2004). However, similar data is currently unavailable for children's responses to continuous versus pulsed tones. Therefore, this study is necessary to explore if children may respond differently in their preference to adults. Indeed, this study has great relevance given that threshold audiometry by air conduction is a widely applied procedure for obtaining auditory thresholds in children, the validity and variability of tonal stimuli presented has not yet been addressed. Indeed, the apparent simplicity of conducting the test often masks the importance of investigating specific procedures applied in pure-tone audiometry. Effective pure-tone test procedures are imperative for the timely and accurate diagnosis of hearing loss in children.

Hearing loss depending on severity, age of onset, and a host of other factors has a mild-to-profound impact on communicative functioning (Yoshinaga-Itano, Sedey, Coulter \& Mehl, 1998). The prevalence of hearing loss in children is such that its diagnosis and management necessitates high prioritisation. It is estimated that in 2002 approximately 72000 school-aged children in the United States of America (U.S.A.) alone fell in the "hearing impairment category" (United States Office of Special Education Programs, 2002). In South Africa, a developing context, prevalence of hearing loss can be expected to be similar or even higher than in the U.S, but to date no statistical data is available for this population. School-aged children with undetected hearing loss that have not received early intervention, often experience academic delays. As would be expected, the primary deficit areas for children with hearing loss are in those subjects that are language-based (Johnson, Benson \& Seaton, 1997). In addition, school-aged children with a hearing loss appear to have increased rates of grade failures, need for educational assistance, and perceived behavioural issues in the classroom (Johnson, Benson \& Seaton, 1997). An undetected hearing loss may have a cascading effect in terms of habilitation. Various effects are insurmountable - such as economic status, education, psychosocial stigma and eventually potential employment opportunities as an adult. Fortunately, there is a continued emphasis nationally and internationally on early identification and monitoring of hearing loss. Therefore, it is vitally important to obtain optimal thresholds for children as these results have direct consequences on identification, diagnosis and management of a hearing loss. Early detection followed by appropriate intervention maximises the benefits to the child, family, and society (Diefendorf, 2002; Pappas, 1998). Part of the intervention process includes the appropriate fitting of hearing aids. However, if optimal and reliable hearing thresholds are not obtained, over or under amplification may occur.

Although more objective tests have been developed as part of the test battery to obtain auditory thresholds, one cannot move away from standard pure-tone audiometry (Hall, 2007). Electrophysiological tests like AEP (auditory evoked potentials) and ECochG (electrocochleography) are used to estimate hearing thresholds. However, these test procedures often require sedation, may have artefact contamination, and are not suitable for all types of losses, e.g. conductive hearing losses or mild sensori-neural hearing losses (Hall, 2007). Electrophysiological tests cannot substitute the use of pure-tone audiometry where reliable behavioural responses can be obtained to determine frequency specific information.

Data regarding the pure-tone testing of school-aged children is of significant importance as this population is mostly evaluated with pure-tone audiometry. School-aged children often pose unique challenges to the audiologist during pure-tone testing (Johnson, et al, 1997). These challenges include assessment of children with developmental delays in, for example, receptive language, which reduces their comprehension and following of test instructions resulting in troublesome test behaviour. Some children will not tolerate earphones or ear inserts for long periods of time as this makes them uncomfortable. In comparison to adults, children have a limited attention span and cannot remain still or concentrate for long periods during testing. This may cause them to become restless and/or uncooperative. False-positive responses are prevalent in this population and failure 
to respond to stimuli close to their hearing thresholds are quite common. In addition, children's reliability of responses tends to decrease as the time of testing increases (Johnson et al, 1997). Therefore, threshold data comparisons for continuous tones and pulsed tones in school-aged children are justified because it may be, for example, that one stimulus tone is perceived as an easier task to respond to, resulting in a shorter testing time. A shorter testing time may address assessment challenges such as reduced attention span and may increase the reliability of responses. Comparisons of continuous and pulsed pure-tone thresholds may yield findings that indicate better thresholds obtained for one of these methods. These findings may guide audiologists when deciding which stimulus tone to use when testing children with pure-tone stimuli.

A review of available professional literature has clearly revealed a lack of data to enable comparisons of continuous tones and pulsed tones in children using the manual puretone technique (as recommended by ASHA, 1978). This exploratory investigation aimed to compare threshold data and listener preference for continuous and pulsed tones on a small group of children. Therefore, the main aim of this study was to compare continuous and pulsed pure-tone audiometry in a group of school-aged children. The subaims of the study were:

- to determine whether a threshold difference existed between continuous versus pulsed pure-tone audiometry for the frequency range of $125 \mathrm{~Hz}$ to $8000 \mathrm{~Hz}$; and

- to determine listener preference for continuous versus pulsed tones for the frequency range of $125 \mathrm{~Hz}$ to 8000 $\mathrm{Hz}$.

\section{Method}

\section{Design}

This exploratory study was mainly quantitative in nature consisting of pure-tone audiometric testing and a brief, three-question interview. A comparative based study employing a quasi-experimental design of research was used as not all variables could be controlled for (Struwig \& Stead, 2001). The main aim of this study was to compare continuous and pulsed pure-tone audiometry in a group of school-aged children.

\section{Participants}

Ethical issues

Ethical clearance was obtained from the departmental research committee of the University of Pretoria before the investigation was carried out. Written informed consent and assent was obtained from the participants and their guardians. Participants and their guardians were informed that their participation was voluntary and that they could withdraw without consequence at any stage during the study. All identifying information was kept confidential and participants were not subjected to harmful or unjust procedures.

\section{Participant selection criteria}

Participants were required to respond reliably and had to concentrate for long periods (40 minutes or longer). For this reason, children between the ages of 8 years to 12 years were selected. It was felt that children in this age group were more able than younger children to perform these tasks consistently.

- Participants had to present with normal hearing as the presence of a hearing loss may influence the ability to distinguish between the continuous and pulsed pure-tone test signal. Furthermore, most researchers are in agreement that when investigating the benefits of any audiological test procedure, normative data should be recorded before similar investigations can be conducted on hearing-impaired populations (Hall, 2007).

- In order to ascertain hearing status, all participants were subjected to a test battery prior to the study. The test battery included otoscopy, tympanometry, reflex testing, oto-acoustic emission screening and comprehensive puretone bone and air conduction testing.

Sampling

Participants were purposefully selected by requesting final year students to supply contact details of family members or acquaintances that had children between the ages of 8 years and 12 years. The participants were expected to visit the Hearing Clinic at the University of Pretoria and therefore only children from the Pretoria region were targeted.

Description of participants

Eighteen children (36 ears) with normal hearing thresholds for all seven tested frequencies were included. According to Katz (2002) and Martin and Clark (2003) a range of $0 \mathrm{~dB}$ to $20 \mathrm{~dB}$ can be regarded as normal hearing for school-aged children. Eight of the participants were male and ten were female. The age distributions were as follows: three were eight years old, one was nine years old, four were ten years old, six were eleven years old and four were twelve years old.

\section{Apparatus and Materials}

\section{Apparatus and material for pre-selection}

Otoscopic examinations were conducted using a WelchAllyn otoscope. Acoustic immittance measures were obtained using a calibrated (according to SABS 0154-1/2, 0182) tympanometer (GSI Tympstar). A Scout Oto-Acoustic Emission machine with $2 \mathrm{kHz}$ to $6 \mathrm{kHz}$ ( $4 \mathrm{~dB}$ to $6 \mathrm{~dB}$ for a pass) screening protocol was used to perform otoacoustic emission screening. Pure-tone air and bone conduction thresholds were obtained using a twin channel diagnostic audiometer, the GSI 61. Participants were seated in a sound-insulated booth (Industrial Acoustics Company, Inc.). Calibration is conducted periodically in accordance with American National Standards Institute (ANSI) 1996 specifications and listening checks of the equipment were performed prior to testing. Results were recorded on a standard audiogram.

Apparatus and material for main study

Pulsed and continuous hearing thresholds were obtained using the same sound-insulated booth and diagnostic audiometer employed for the pre-selection of subjects. An adaptation of a standard audiogram was used to record the results. The symbol that was used for both ears to indicate continuous hearing threshold level was an outline of a circle and a solid circle indicated pulsed hearing thresholds. A brief three-question interview schedule was used after completion of the hearing test to obtain information about listener preference for the type of test signal.

Procedures

Pure-tones were presented through earphones to assess air conduction thresholds. The results of the air conduc- 
tion tests in both test conditions (continuous and pulsed) were plotted on an audiogram from which the results were compared. The ascending/descending method developed by Carhart and Jerger (1959) was used to obtain pure-tone thresholds bilaterally at $125 \mathrm{~Hz}, 250 \mathrm{~Hz}, 500 \mathrm{~Hz}, 1000 \mathrm{~Hz}$, $2000 \mathrm{~Hz}, 4000 \mathrm{~Hz}$, and $8000 \mathrm{~Hz}$. Octave frequencies of $3000 \mathrm{~Hz}$ and $6000 \mathrm{~Hz}$ were excluded to reduce testing time. However, these octaves were included in the initial selection procedures to determine normal criteria. Steps of $5 \mathrm{~dB}$ were used to confirm hearing threshold levels. Participants took part in one testing session with two alternating signal conditions. The signal conditions were a manually presented continuous tone for one second to two seconds and two short pulses (rise-fall time of $35 \mathrm{~ms}$ with a duration of $200 \mathrm{~ms}$ onset to offset) automatically generated by the audiometer. The test signals were presented in an alternating manner to ensure that the same test signal (e.g. a pulsed pure tone) was not always presented in the same order to the particpant. For example, the participant would hear, at $125 \mathrm{~Hz}$, first a pulsed then a continuous tone. At $250 \mathrm{~Hz}$, he/she would first hear a continuous then a pulsed tone. This would randomly vary from participant to participant. This presentation ensured reliability and validity of results and yielded two sets of thresholds (continuous and pulsed tones) for each ear. The first signal (either automatically pulsed or a continuous tone) was randomly chosen and followed alternately by the other signal. After the hearing test, a brief three-question interview followed the pure-tone testing. Subjects were asked if they preferred one test signal above the other, and, if so, which signal they preferred and why. Their answers were recorded by the researcher on a space provided on the audiogram. Testing and recording was done by a final year audiology student. A qualified audiologist supervised the student during testing of the first three subjects to ensure that test procedures were valid and reliable. The supervisor was available for consultation for the duration of the testing.

Data analysis

Data was organised and processed using "Microsoft Office Excel" software. A univariate procedure was used in order to draw comparisons between continuous and pulsed threshold values at discreet frequencies. The average threshold, mean difference and standard deviation of the two thresholds obtained with the two different types of stimuli at each frequency tested for each ear, was calculated. Listeners' preferences indicated in the interview were calculated in percentages to obtain the most preferred signal. Reasons for preferring one signal to another was analysed qualitatively. During data analysis the results for each ear were not combined as literature suggests that ear differences occur (Hochberg \& Waltzman, 1972). The afore-mentioned statistical procedures used in the study were deemed appropriate for the small sample size and provided sufficient data in order to answer the research question comprehensively.

Results

Determining whether a threshold difference exists between continuous versus pulsed pure-tone audiometry

The first sub-aim of the study was to determine whether a threshold difference exists between continuous versus pulsed pure-tone audiometry in school-aged children.
These results included hearing threshold levels obtained for continuous as well as pulsed tones at frequencies $125 \mathrm{~Hz}$ to $8000 \mathrm{~Hz}$ in each ear of each participant. The difference between the average of the continuous hearing thresholds and the average of the pulsed hearing thresholds for each ear and specific frequencies were determined. These differences were calculated by subtracting the averaged pulsed tones from the averaged continuous tones and are depicted in Figure 1.

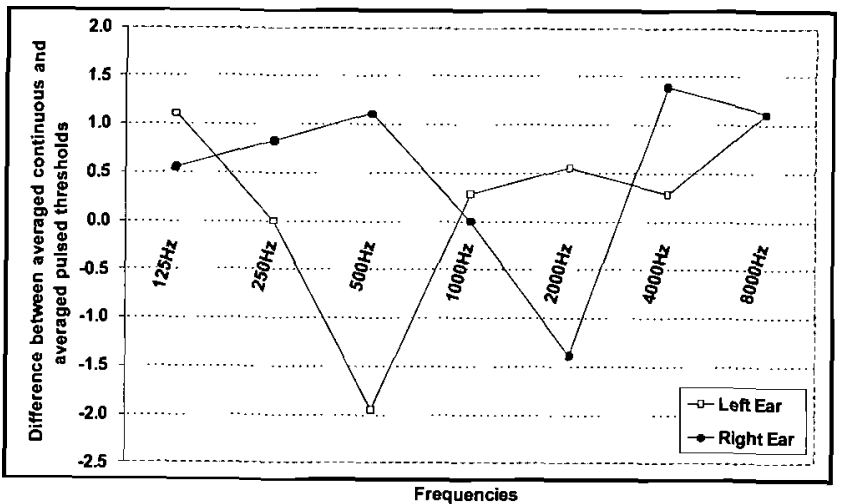

Figure 1: Difference between average bearing threshold levels of continuous and pulsed tones in each ear $(n=18)$

A difference value of zero, which was obtained at 250 $\mathrm{Hz}$ in the left ear and at $1000 \mathrm{~Hz}$ in the right ear, indicated no threshold difference between the two stimulus types as presented in Figure 1. Negative values indicated that lower thresholds were obtained for continuous tones. Continuous tones yielded better thresholds than pulsed tones at $500 \mathrm{~Hz}$ in the left ear and $2000 \mathrm{~Hz}$ in the right ear. Lower thresholds were obtained for pulsed tones at all other frequencies for both ears. Therefore, pulsed tones yielded lower thresholds than continuous tones for the majority of frequencies tested.

In order to determine whether the signal type yielded a threshold difference at specific frequencies, findings per frequency for the respective ears were analysed. The standard deviation and the mean average of the hearing threshold levels obtained at each frequency tested, for each stimulus type, for the left ear, follows in Figure 2.

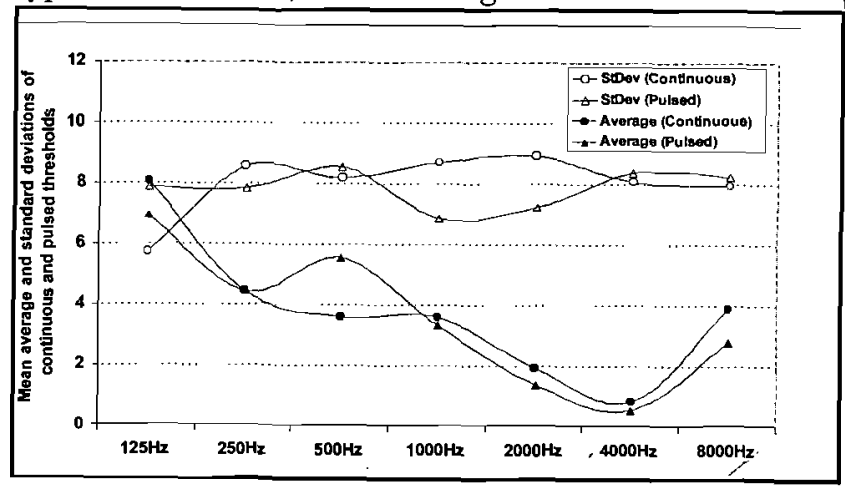

Figure 2: Mean average and standard deviation for continuous and pulsed tones in the left ear $(n=18)$

The average standard deviation for continuous tones was $8 \mathrm{~dB}$ in the left ear. The average standard deviation for pulsed tones was $7.9 \mathrm{~dB}$ in the left ear. Continuous tones 
presented less variation in thresholds at $125 \mathrm{~Hz}, 500 \mathrm{~Hz}$, $4000 \mathrm{~Hz}$ and $8000 \mathrm{~Hz}$ in the left ear while pulsed tones presented less variation in thresholds at $250 \mathrm{~Hz}, 1000 \mathrm{~Hz}$ and $2000 \mathrm{~Hz}$. Absolute differences in averaged thresholds at each test frequency ranged from $0 \mathrm{~dB}$ to $1.9 \mathrm{~dB}$ between $125 \mathrm{~Hz}$ and $8000 \mathrm{~Hz}$. Averaged pulsed tones yielded slightly lower thresholds at all frequencies except at $250 \mathrm{~Hz}$ where thresholds were equal and at $500 \mathrm{~Hz}$ where the continuous tones revealed slightly lower thresholds.

Similar to the left ear, findings per frequency were analysed for the right ear to determine whether the signal type yielded a threshold difference at specific frequencies. The standard deviation and the mean average of the hearing threshold levels were obtained at each frequency tested, for each stimulus type, for the right ear, follows in Figure 3.

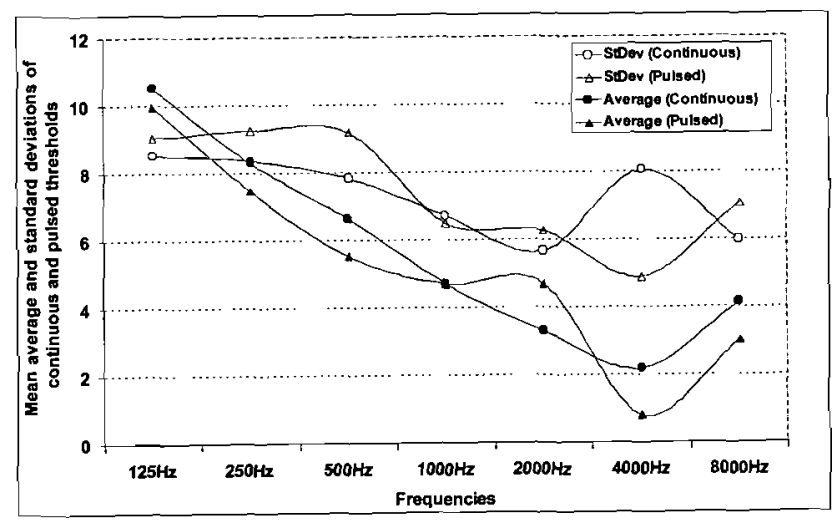

Figure 3: Mean averages and standard deviation for continuous and pulsed tones in the right ear $(n=18)$.

The average of the standard deviations for continuous tones was $7.3 \mathrm{~dB}$ compared to $7.5 \mathrm{~dB}$ for pulsed tones in the right ear. Continuous tones presented less variation in all thresholds except at $1000 \mathrm{~Hz}$ and $4000 \mathrm{~Hz}$ in the right ear. Absolute differences in averaged thresholds at each test frequency ranged from $0 \mathrm{~dB}$ to $1.4 \mathrm{~dB}$ between 125 $\mathrm{Hz}$ and $8000 \mathrm{~Hz}$. Averaged pulsed tones yielded slightly lower thresholds at all frequencies except at $1000 \mathrm{~Hz}$ where thresholds were equal and $2000 \mathrm{~Hz}$ where the continuous tones obtained slightly lower thresholds.

When results of both ears are considered, threshold standard deviations (averaged over all frequencies tested) showed that the pulsed presentation method yielded slightly less variation $(0.005 \mathrm{~dB})$ in thresholds, than the continuous presentation method. On average, pulsed tones yielded slightly lower thresholds where the average pulsed tone threshold was $0.4 \mathrm{~dB}$ lower than for the averaged continuous tone stimulus. These small differences in averaged thresholds and standard deviations obtained for both test tones reinforce the equality of the two stimulus signals. Based on the aforementioned, the overall differences would not be sufficient to recommend one signal above the other.

Findings for both ears at all frequencies were plotted to compare overall threshold differences due to different signals. These results are presented in Figure 4.

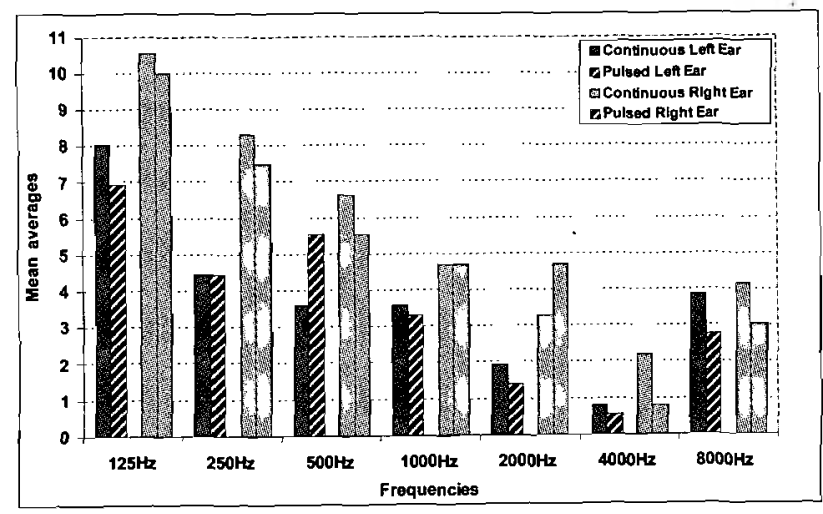

Figure 4: $A$ comparison of the average bearing thresholds obtained for continuous and pulsed tones in each ear $(n=18)$.

As may be noted in Figure 4, the differences in thresholds between the two stimuli were relatively small. This correlates with results obtained by Hochberg and Waltzman (1972), which indicated that both types of signals obtained comparable threshold levels at all audiometric frequencies tested, and did not vary by more than $1.6 \mathrm{~dB}$. Across all frequencies tested in the left ear, an average of $3.8 \mathrm{~dB}$ for continuous tones and $3.6 \mathrm{~dB}$ for pulsed tones was obtained. Similarly, the average hearing thresholds obtained in the right ear were $5.7 \mathrm{~dB}$ for continuous tones and $5.2 \mathrm{~dB}$ for pulsed tones. Although the automatically pulsed tone threshold (averaged across the frequencies tested) was lower than for the continuous tone, the average difference was only $0.2 \mathrm{~dB}$ in the left ear and $0.5 \mathrm{~dB}$ in the right ear. This small difference is not important in clinical applications for which $5 \mathrm{~dB}$ increments are used in pure-tone audiometry.

Taken as a whole, the pulsed tone stimuli produced slightly lower thresholds at all the test frequencies where the average pulsed tone threshold was $0.3 \mathrm{~dB}$ lower than for the continuous tone stimulus. This differs from the study done on adults where lower thresholds (averaged across all frequencies) were obtained for continuous tones than for automatically pulsed tones, where the difference was $1.0 \mathrm{~dB}$ (Burk \& Wiley, 2004). The obtained minor $0.3 \mathrm{~dB}$ difference would not result in a substantial difference in hearing threshold level, since these measurements are made in $5 \mathrm{~dB}$ increments.

Determining whether a listener preference is perceived between continuous versus pulsed tones

The second aim of the study was to determine whether participants had a listener's preference for one of the two signals. The percentage of participants preferring one signal over the other based on the three questions asked, was calculated. These results are represented Figure 5. 


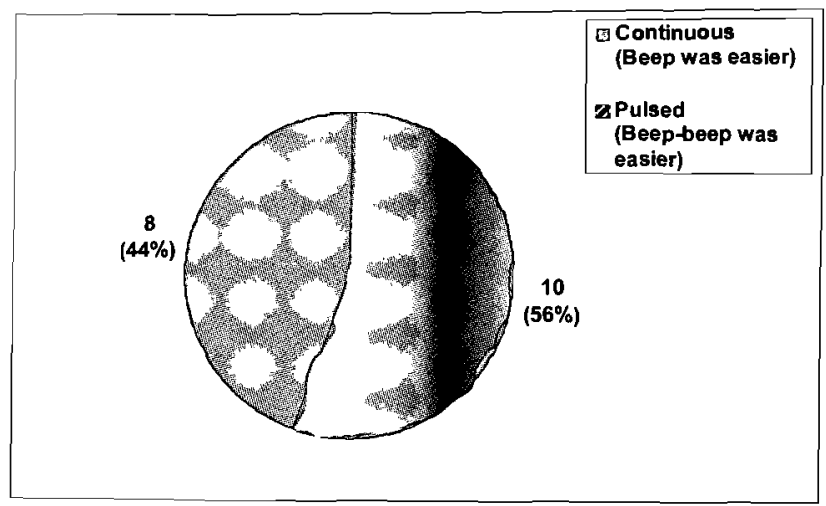

Figure 5: Listeners' preference for one stimulus type over the other $(n=18)$.

The difference in the percentage of perceived preference was $11 \%$, with $56 \%$ preferring continuous tones and $44 \%$ preferring pulsed tones. This differs from the findings of a study (Burk \& Wiley, 2004) done on 24 adults, where it was found that $62 \%$ of subjects preferred the pulsed tones and $38 \%$ preferred the continuous tones. Over two-thirds of normal and tinnitus subjects, in a study done by Hochberg and Waltzman (1972), preferred to listen to pulsed tones as opposed to continuous tones during threshold determination. The difference in the sample population group in the present study, which was done with children, may contribute to the disparity in the findings.

Results indicated that more participants preferred the continuous tone, although, the post-test comments across the signal types were very similar. This differs from previous investigations where persons experiencing tinnitus commented that continuous tones are more confusing than pulsed tones (Mineau \& Schlauch, 1997). Findings revealed that the most common explanation given by those participants preferring the continuous tones was that it was louder and clearer and that it did not have interruptions. The most common reason given by those participants preferring the pulsed tones was that one could hear it a second time if one was unsure of hearing the first presentation.

A direct comparison provided further findings regarding the correlation between the perceived listener's preference and the stimulus signal that produced the lower hearing threshold level. Seven subjects obtained lower hearing thresholds in both ears for the stimulus signal that was not their perceived preference. A further seven subjects perceived preference correlated with a lower hearing threshold in one ear only. Only four participants obtained better hearing thresholds in both ears for the stimulus signal that they perceived as their preference. Therefore, it would seem that listeners' perceived preference did not correlate with a lower hearing threshold for the preferred stimulus signal.

\section{- Discussion and Conclusion}

The aim of this study was to compare continuous and pulsed pure-tone audiometry in a group of normal-hearing school-aged children. This was done by comparing hearing threshold levels obtained for both stimulus signals and determining if a listener's preference existed for one tone over the other. The value of this study, was that data, unlike previous studies of the same nature (Burk \& Wiley, 2004;
Mineau \& Schlauch, 1997), was recorded and analysed separately for each ear at the various test frequencies.

In summary, the findings obtained for the averaged hearing threshold differences at each frequency when comparing continuous to pulsed hearing threshold levels were:

- Pulsed tones yielded slightly lower thresholds in both ears at $125 \mathrm{~Hz}$.

At $250 \mathrm{~Hz}$, no difference in hearing threshold levels were obtained in the left ear and pulsed tones yielded slightly lower thresholds in the right ear.

- At $500 \mathrm{~Hz}$, continuous tones yielded slightly lower thresholds in the left ear and pulsed tones yielded slightly lower thresholds in the right ear.

At $1000 \mathrm{~Hz}$, pulsed tones yielded slightly lower thresholds in the left ear and no difference in hearing threshold levels were obtained in the right ear.

- At $2000 \mathrm{~Hz}$, pulsed tones yielded slightly lower thresholds in the left ear and continuous tones yielded slightly lower thresholds in the right ear.

- Pulsed tones yielded slightly lower thresholds in both ears at $4000 \mathrm{~Hz}$.

- Pulsed tones yielded slightly lower thresholds in both ears at $8000 \mathrm{~Hz}$.

The above-mentioned findings revealed that the pulsed tone stimuli produced slightly lower thresholds $(0.3 \mathrm{~dB})$ on average at all the test frequencies than for the continuous tone stimulus. Literature does not provide an explanation for why audiometric pulsed tones yield slightly lower thresholds, except that subjects tend to report that a pulsed tone seems to be more alerting than a continuous tone ( $\mathrm{Mc}^{-}$ Commons \& Hodge, 1969). Inter-aural differences were apparent at some test frequencies in this investigation and are also documented (Hochberg \& Waltzman, 1972). The ear that produced the better pulsed tone thresholds varied within the same subject, which makes the theory of ear domination invalid for these cases. Current literature does not aid in providing an explanation nor are the results of this study useful to explain why these inter-aural differences are found for continuous and pulsed tone thresholds. However, differences are statistically so small that it does not warrant speculation as to why they occur.

It may be concluded that the use of pulsed tones for audiometric threshold measures in normal-hearing children had no clinically significant effect on obtained hearing thresholds as $5 \mathrm{~dB}$ increments are used in pure-tone audiometric testing. This differs from previous findings demonstrating the benefits of using pulsed tones in threshold assessment for adults with sensorineural hearing loss and persons with tinnitus (Burk \& Wiley, 2004; Hochberg \& Waltzman, 1972; Mineau \& Schlauch, 1997). These preliminary findings may indicate that school-aged children yield different responses during pure-tone testing than adults.

However, continuous tones showed a slight advantage in terms of presenting what is perceived as an easier task without adversely affecting the test's outcome. A slight difference was obtained, with more participants preferring continuous tones than pulsed tones. The slight preference for continuous tones in normal-hearing children also differs from the study done on adults, where the preference was for pulsed tones (Burk \& Wiley, 2004). In this study, it was also found 


\section{CONTINUOUS VERSUS PULSED PURE-TONE AUDIOMETRY IN A GROUP OF SCHOOL-AGED CHILDREN}

that, overall, listeners' perceived preference did not correlate with a lower hearing threshold for the preferred stimulus signal. Reasons for these findings are unexplained in literature to date.

\section{Limitations}

The study presented limitations in terms of sample size and ages included in the selected age range. In addition, as with most quasi-experimental studies, not all variables could be controlled for such as tester-bias and the participants' attention span. Although, the person who conducted the testing was the same person who analysed the data, the researcher ensured that data was recorded and analysed as accurately and objectively as possible through supervision. The person conducting the testing reported that all participants demonstrated sufficient attention span for the duration of the test procedure. She indicated that, although some subjects showed obvious signs of boredom, this resulted in only a few inconsistent responses and not more than one would expect during behavioural testing. Despite these shortcomings, limited research is available to date that compares outcomes of different test signals in pure-tone audiometry in children and this exploratory study provides preliminary data with some clinical considerations for audiologists in practice.

\section{Clinical Implications}

The benefits of using pulsed tones over continuous tones have been documented with adults with hearing-loss as well as with adults suffering from tinnitus. However, in this exploratory study it would seem that using either continuous or pulsed pure-tone signals during testing with normalhearing children does not yield significant threshold differences. Conversely, audiologists might consider asking their young clients during testing which signal they preferred. As revealed in this study, this may not result in obtaining better threshold levels. Nevertheless, taking their preference into consideration may improve cooperation and make the testing experience less strenuous for the young client.

\section{Recommendations}

A future study could include a larger sample and smaller age intervals with more subjects per age interval to ensure more generalizability of findings. This would also allow for inter variable analysis on the influence of gender and auditory maturation oniresults. In addition, a larger sample may yield results that are more directly comparable to similar international studies on adults.

\section{References}

American National Standards Institute. (1996). Specifications for Audiometers, S3.6. New York: ANSI.

American Speech-Language-Hearing Association. (1978). Guidelines for Manual Pure-tone Audiometry. Asha, 20, 279-301.

Blandy, S. \& Lutman, M. (2005). Hearing threshold levels and speech recognition in noise in 7-year-olds. International Journal of Audiology, 44, 435-443.

Burk, M.H. \& Wiley, T.L. (2004). Continuous versus pulsed tones. American Journal of Audiology, 13, 54-61.

Carhart, R. \& Jerger, J. (1959). Preferred methods of determination of pure-tone threshold. In J.W. Hall \& H.G. Meuller (Eds.). Audiologists' Desk Reference. San Diego: Singular.

Dancer, J.E. \& Conn, M. (1983). Effects of two procedural modi- fications of the frequency of false-alarm responses during puretone threshold determination. Journal of Auditory Research, 23, 215-219.

Dancer, J., Ventry, I.M. \& Hill, W. (1976). Effects of stimulus presentation and instructions on pure-tone thresholds and falsealarm responses. Journal of Speech and Hearing Disorders, 41, 315-324.

Diefendorf, A.O. (2002). Detection and assessment of hearing loss in infants and children. In J. Katz (ed.). Handbook of Clinical $A u-$ diology (5th Ed.). Baltimore: Lippincott Williams \& Wilkins.

DiGiovanni, J.J. \& Repka, J.N. (2007). Response method in audiometry. American Journal of Audiology, 16, 145-148.

Gardner, M.B. (1947). A pulsed-tone technique for clinical audiometric threshold measurements. The Journal of the Acoustical Society of America, 19, 592-599.

Hall, J.W. (2007). New bandbook of auditory evoked responses. Boston: Pearson Edcuation.

Hallpike, C.S. \& Hood, J.D. (1951). Some recent work on auditory adaptation and its relationship to the loudness recruitment phenomenon. The Journal of the Acoustical Society of America, 23, 270-274.

Hochberg, I. \& Waltzman, S. (1972). Comparison of pulsed and continuous tone thresholds in clients with tinnitus. Audiology, 11, 337-342.

Hood, J.D. (1955). Auditory fatigue and adaptation in the differential diagnosis of end-organ disease. Annals of Otology, Rhinology and Laryngology, 64, 507-518.

Jerger, J. (1960). Bekesy Audiometry in analysis of auditory disorders. Journal of Speech and Hearing Research, 3, 275-287.

Johnson, C.D., Benson, P.V., \& Seaton, J.B. (1997). Educational Audiology Handbook. San Diego: Singular.

Katz, J. (2002). Handbook of Clinical Audiology. (5 $5^{\text {th }}$ Ed.). Baltimore: Lippincott Williams \& Wilkins.

McCommons, R.B. \& Hodge, D.C. (1969). Comparison of continuous and pulsed tones for determining Bekesy threshold measurements. The Journal of the Acoustical Society of America, 45, 1499-1504.

Margolis, R.H. \& Saly, G.L. (2007). Towards a standard description of hearing loss. International Journal of Audiology, 46, 746758.

Martin, F.N. \& Clark, J.G. (2003). Introduction to Audiology. (8 ${ }^{\text {th }}$ Ed.). Boston: Pearson Education.

Martin, F. \& Sides, D.G. (1985). Survey of current audiometric practice. Asha, 27, 29-36.

Mineau, S.M. \& Schlauch, R.S. (1997). Threshold measurement for clients with tinnitus: Pulsed or continuous tones. American Journal of Audiology, 6, 52-56.

Newby, H.A. \& Popelka, G.R. (1985). Audiology. (5 $5^{\text {th }}$ Ed.). New Jersey: Prentice-Hall.

Pappas, D.G. (1998). Diagnosis and Treatment of Hearing Impairment in Children. ( $2^{\text {nd }}$ Ed.). San Diego: Singular.

Reger, S.N. (1970). Bekesy audiometry and the method of limits. International Audiology, 9, 24-29.

Silman, S. \& Silverman, C.A. (1991). Auditory Diagnosis: Principles and Applications. San Diego: Academic Press.

Sorensen, H. (1962). Clinical application of continuous threshold recording. Acta Otolaryngology, 54, 403-422.

Struwig, F.W. \& Stead, G.B. (2001). Planning, Designing and Reporting Research. South Africa: Pearson Education.

United States Office of Special Education Programs. (2002). 


\section{CATHERINE VAN DIJK AND NAEEMA OSMAN}

Child count: Number served (ages 6-21), by disability and state. Yoshinaga-Itano, C., Sedey, A.L., Coultier, D.K., \& Mehl, A.L. Retrieved July 22, 2006, from http://www.ideadata.org/tables26th/ar_aa3.htm. (1998). Language of early- and later- identified children with hearing loss. Pediatrics, 102, 1161-1171. 